Proc. of the 11 Int. School on Theoretical Physics Symmetry and Structural Properties of Condensed Matter, Rzeszów 2014

\title{
Spin and Charge Transport in a Magnetic Tunnel Junction with Magnetic Impurities Embedded in the Tunnel Barrier
}

\author{
M. MisIORNY ${ }^{a, b, c, *}$ AND J. BARNAS $\bar{S}^{c, d}$ \\ ${ }^{a}$ Department of Microtechnology and Nanoscience (MC2), Chalmers Univeristy of Technology, \\ S-41296 Göteborg, Sweden \\ ${ }^{b}$ Peter Grünberg Institut PGI-2, Forschungszentrum Jülich, D-52425 Jülich, Germany \\ ${ }^{c}$ Faculty of Physics, Adam Mickiewicz University, PL-61614 Poznań, Poland \\ ${ }^{d}$ Institute of Molecular Physics, Polish Academy of Sciences, PL-60179 Poznań, Poland
}

\begin{abstract}
Electronic transport in a nanoscopic magnetic tunnel junction with magnetic particles or magnetic impurity atoms/molecules embedded in the barrier is studied theoretically. The impurity Hamiltonian includes magnetic anisotropy of easy axis type with additional perpendicular term. The description takes into account both elastic tunneling processes as well as inelastic processes associated with a flip of electron spin.
\end{abstract}

DOI: 10.12693 /APhysPolA.128.196

PACS: 72.10.Fk, 72.25.-b, 73.23.-b, 75.50.Xx

\section{Introduction}

Magnetic tunnel junctions (MTJs) consisting of two thin layers of a ferromagnetic metal and separated by an ultrathin layer of insulating material exhibit a tunnel magnetoresistance (TMR) effect, associated with the change of magnetic configuration from parallel to antiparallel alignment [1]. Magnitude of the effect significantly depends on the insulating barrier. A very large TMR ratio has been found in MTJs with epitaxial $\mathrm{MgO}$ barriers [2]. In the later case, however, the TMR effect cannot be accounted for by the simple Julliere model, and the large TMR results rather from specific spin-filtering properties of the epitaxial $\mathrm{MgO}$ barrier [3]. Owing to the large TMR ratio, the MTJs with MgO tunnel barriers are considered currently as highly promising systems for various applications in spintronics devices and also in the information technology $[4,5]$.

When a barrier is crystalline, and the interfaces between the barrier and electrodes are structurally perfect, electrons transmitted through the barrier conserve their wave vector component parallel to the barrier plane. However, tunnel barriers are usually not defect-free and contain a number of impurities and other structural defects. Here we consider the situation when the tunnel barrier includes magnetic impurities/nanoparticles in the following referred to also as magnetic impurities. Electrons tunneling through the barrier can interact with the impurities, which leads to reversal of the electron spin. This, in turn, leads to a change in the magnetic state of these impurities. The main objective of this paper is a theoretical analysis of the influence of magnetic impurities in the barrier on the charge and spin currents

* corresponding author; e-mail: misiorny@amu.edu.pl due to electron tunneling. We consider a nanoscopic junction with a single magnetic impurity in the barrier.

\section{Model}

The total Hamiltonian $\mathrm{H}$ of the junction consists of three terms,

$$
\widehat{\mathcal{H}}=\widehat{\mathcal{H}}_{\mathrm{imp}}+\widehat{\mathcal{H}}_{\mathrm{el}}+\widehat{\mathcal{H}}_{\mathrm{T}},
$$

which describe the magnetic impurity, two external electrodes, and electron tunneling through the barrier, respectively. We assume that the highest occupied and lowest unoccupied levels of the magnetic impurity are sufficiently far from the Fermi level, so they do not take part in transport for bias voltages of interest. Thus, the charge state of the impurity is not changed when junction is biased, and electrons tunneling through the barrier can interact with the impurity by exchange interaction only. Moreover, we assume that the magnetic impurity in an unbiased junction is described by a spin operator $\widehat{\boldsymbol{S}}$, and the corresponding spin number $S$ is also conserved. Thus, the impurity can be described by the giant-spin Hamiltonian $[6,7]$ :

$$
\widehat{\mathcal{H}}_{\mathrm{imp}}=-D \widehat{S}_{z}^{2}+(E / 2)\left(\widehat{S}_{+}^{2}+\widehat{S}_{-}^{2}\right) \text {, }
$$

where the first and second terms describe the uniaxial and transverse magnetic anisotropy, respectively, with $D$ and $E$ denoting the corresponding anisotropy constants, and $\widehat{S}_{ \pm}=\widehat{S}_{x} \pm \mathrm{i} \widehat{S}_{y}$ being the spin ladder operators.

In the single-electron approximation, ferromagnetic electrodes can be described by the Hamiltonian

$$
\widehat{\mathcal{H}}_{\mathrm{el}}=\sum_{q \boldsymbol{k} \sigma} \varepsilon_{\boldsymbol{k} \sigma}^{q} \widehat{a}_{\boldsymbol{k} \sigma}^{q \dagger} \widehat{a}_{\boldsymbol{k} \sigma}^{q},
$$

where $\varepsilon_{\boldsymbol{k} \sigma}^{q}$ is the single electron energy in the $q$-th electrode $(q=L$ for the left electrode, and $q=R$ for the right one), $\boldsymbol{k}$ stands for a wave vector, and $\sigma$ is the electron spin index. Furthermore, $\widehat{a}_{\boldsymbol{k} \sigma}^{q \dagger}\left(\widehat{a}_{\boldsymbol{k} \sigma}^{q}\right)$ is the creation (annihilation) operator of an electron in the $q$-th 
electrode. Both electrodes are characterized by a spindependent density of electron states, $\rho_{\sigma}^{q}(\varepsilon)$. In turn, magnetic properties of the $q$-th electrode can be described by the corresponding spin polarization factor $p_{q}$, defined by densities of states at the Fermi level $\mu_{0}$ as $p_{q}=\left[\rho_{\uparrow}^{q}\left(\mu_{0}\right)-\rho_{\downarrow}^{q}\left(\mu_{0}\right)\right] /\left[\rho_{\uparrow}^{q}\left(\mu_{0}\right)+\rho_{\downarrow}^{q}\left(\mu_{0}\right)\right]$.

In the framework of an effective tunneling Hamiltonian, electron transport between the electrodes can be described by the Hamiltonian [8],

$$
\begin{aligned}
& \widehat{\mathcal{H}}_{\text {tun }}=t \sum_{\boldsymbol{k} \boldsymbol{k}^{\prime}}\left\{\alpha_{\mathrm{d}} \sum_{q \alpha} \widehat{a}_{\boldsymbol{k} \alpha}^{q \dagger} \widehat{a}_{\boldsymbol{k}^{\prime} \alpha}^{\bar{q}}\right. \\
& \left.\quad+\sum_{q q^{\prime}} \alpha_{\mathrm{ex}}^{q q^{\prime}} \sum_{\alpha \beta}\left(\boldsymbol{\sigma}_{\alpha \beta} \cdot \widehat{\boldsymbol{S}}\right) \widehat{a}_{\boldsymbol{k} \alpha}^{q \dagger} \widehat{a}_{\boldsymbol{k}^{\prime} \beta}^{q^{\prime}}\right\},
\end{aligned}
$$

where $\boldsymbol{\sigma} \equiv\left(\sigma_{x}, \sigma_{y}, \sigma_{z}\right)$ is the vector consisting of the Pauli matrices. The first term of Eq. (3) represents direct tunneling between the electrodes (no interaction with impurity), while the second term describes tunneling processes in which electrons interact via exchange coupling with the magnetic impurity. Strength of the former processes is described by the product $K \alpha_{d}$, whereas of the latter ones by $K \alpha_{\mathrm{ex}}^{q q^{\prime}}$. It is convenient to write $\alpha_{\mathrm{ex}}^{q q^{\prime}}$ in the form $\alpha_{\mathrm{ex}}^{q q^{\prime}} \equiv \nu_{q} \nu_{q^{\prime}} \alpha_{\mathrm{ex}}$, where a dimensionless parameter $\nu_{q}$ quantifies the coupling of the magnetic impurity to the $q$-th electrode. Thus, $K$ is the key barrier parameter $[9,10]$, whereas $\alpha_{\mathrm{ex}} / \alpha_{\mathrm{d}}$ describes relative strength of direct electron tunneling and tunneling with interaction between the electrons and impurity.

Without loss of generality, we assume that $E \geq 0$. If $E=0$, then the eigenstates of the magnetic impurity coincide with the eigenstates $|m\rangle$ of the $\widehat{S}_{z}$ operator. On the other hand, the transverse anisotropy $(E \neq 0)$ leads to mixing of the states corresponding to different $m$. As a result, each of the $2 S+1$ eigenstates $|\chi\rangle$ of the Hamiltonian (1), $\widehat{\mathcal{H}}_{\text {imp }}|\chi\rangle=E_{\chi}|\chi\rangle$, is a linear combination of the eigenstates $|m\rangle$. Moreover, note that the eigenstates $|\chi\rangle$ are nondegenerate for integer $S$, while a twofold degeneracy (the Kramers doublets) survives for half-integer $S$ [11].

\section{Transport characteristics}

We assume weak coupling between the electrodes and the impurity. Charge and spin transport can be then described within the approach based on the corresponding master equation. Detailed balance of electrons $(e<0)$ tunneling out/to each electrode allows for writing the charge current as $I_{\mathrm{c}}=\left(I_{\mathrm{c}}^{\mathrm{L}}-I_{\mathrm{c}}^{\mathrm{R}}\right) / 2$, with

$$
I_{\mathrm{c}}^{q}=e \sum_{\boldsymbol{k} \boldsymbol{k}^{\prime}} \sum_{\sigma \sigma^{\prime}} \sum_{\chi \chi^{\prime}} \mathcal{P}_{\chi}\left\{\Gamma_{\left|\bar{q} \boldsymbol{k}^{\prime} \sigma^{\prime}, \chi^{\prime}\right\rangle}^{\mid q \boldsymbol{k} \sigma, \chi}-\Gamma_{\left|q \boldsymbol{k}^{\prime} \sigma^{\prime}, \chi^{\prime}\right\rangle}^{\mid \bar{q} \boldsymbol{k} \sigma, \chi}\right\},
$$

and the spin current as $I_{\mathrm{S}}=\left(I_{\mathrm{s}}^{L}-I_{\mathrm{s}}^{R}\right) / 2$, with

$$
\begin{aligned}
I_{\mathrm{S}}^{q} & =\frac{\hbar}{2} \sum_{q^{\prime}} \sum_{\boldsymbol{k} \boldsymbol{k}^{\prime}} \sum_{\sigma} \sum_{\chi \chi^{\prime}} \mathcal{P}_{\chi}\left\{\Gamma_{\left|q^{\prime} \boldsymbol{k}^{\prime} \sigma, \chi^{\prime}\right\rangle}^{\mid q \boldsymbol{k} \uparrow}-\Gamma_{\left|q \boldsymbol{k}^{\prime} \uparrow, \chi^{\prime}\right\rangle}^{\left|q^{\prime} \boldsymbol{k} \sigma, \chi\right\rangle}\right. \\
& \left.-\left[\Gamma_{\left|q^{\prime} \boldsymbol{k}^{\prime} \sigma, \chi^{\prime}\right\rangle}^{|q \boldsymbol{k} \downarrow, \chi\rangle}-\Gamma_{\left|q \boldsymbol{k}^{\prime} \downarrow, \chi^{\prime}\right\rangle}^{\left|q^{\prime} \boldsymbol{k} \sigma, \chi\right\rangle}\right]\right\},
\end{aligned}
$$

where $\Delta_{\chi \chi^{\prime}}=\varepsilon_{\chi}-\varepsilon_{\chi^{\prime}}$, and

$$
\Gamma_{\left|q^{\prime} \boldsymbol{k}^{\prime} \sigma^{\prime}, \chi^{\prime}\right\rangle}^{|q \boldsymbol{k} \sigma, \chi\rangle}=W_{\left|q^{\prime} \boldsymbol{k}^{\prime} \sigma^{\prime}, \chi^{\prime}\right\rangle}^{|\boldsymbol{k} \sigma, \chi\rangle} f_{q \sigma}\left(\varepsilon_{\boldsymbol{k} \sigma}^{q}\right)\left[1-f_{q^{\prime} \sigma^{\prime}}\left(\varepsilon_{\boldsymbol{k}^{\prime} \sigma^{\prime}}^{q^{\prime}}\right)\right] .
$$

In the above equations $\mathcal{P}_{\chi}$ is the probability of finding the magnetic impurity in the state $|\chi\rangle$, and $f_{q \sigma}(\varepsilon)=[1+$ $\left.\exp \left(\left(\varepsilon-\mu_{\sigma}^{q}\right) / k_{\mathrm{B}} T\right)\right]^{-1}$ is the Fermi-Dirac distribution function, with $T$ denoting the temperature of the system. Moreover, we use the notation $|q \boldsymbol{k} \sigma, \chi\rangle \equiv|q \boldsymbol{k} \sigma\rangle_{\mathrm{el}} \otimes|\chi\rangle_{\mathrm{imp}}$ for the full state of the system. In turn, the transition rates $W_{|j\rangle}^{|i\rangle}$ in the Fermi golden rule approximation are given by

$$
W_{|j\rangle}^{|i\rangle}=\frac{2 \pi}{\hbar}\left|\left\langle j\left|\widehat{\mathcal{H}}_{\text {int }}\right| i\right\rangle\right|^{2} \delta\left(E_{j}-E_{i}\right),
$$

where $|i\rangle$ and $|j\rangle$ are the initial and final states of the system, respectively, while $E_{i}$ and $E_{j}$ denote the corresponding energies. Finally, the spin-dependent electrochemical potential of the $q$-th electrode can be written as $\mu_{\sigma}^{q}=\mu_{0}+e \eta_{q}\left(\delta V+\eta_{\sigma} \delta V_{\mathrm{s}}\right) / 2$, where $\eta_{\mathrm{L}(\mathrm{R})} \equiv \pm 1$, $\eta_{\uparrow(\downarrow)}= \pm 1$, while $\delta V$ and $\delta V_{\mathrm{s}}$ represent the voltage and spin bias, respectively.

Equations (4) and (5) involve both single-electrode $\left(q=q^{\prime}\right)$ and two-electrode $\left(q \neq q^{\prime}\right)$ processes. In order to make use of these equations one also needs to know the probabilities of finding the magnetic impurity in the state $|\chi\rangle$, which can be determined from a set of the stationary master equations

$$
\sum_{\chi^{\prime}} \sum_{q q^{\prime}}\left\{\mathcal{P}_{\chi^{\prime}} \gamma_{\chi^{\prime} \chi}^{q q^{\prime}}-\mathcal{P}_{\chi} \gamma_{\chi \chi^{\prime}}^{q q^{\prime}}\right\}=0
$$

for each $\chi$, with the probability normalization condition $\sum_{\chi} \mathcal{P}_{\chi}=1$. The transition rates $\gamma_{\chi \chi^{\prime}}^{q q^{\prime}}$ between two different impurity states $|\chi\rangle$ and $\left|\chi^{\prime}\right\rangle$, which accompany tunneling of a single electron between the electrodes $q$ and $q^{\prime}$, are given by $\gamma_{\chi \chi^{\prime}}^{q q^{\prime}}=\sum_{\boldsymbol{k} \boldsymbol{k}^{\prime}} \sum_{\sigma \sigma^{\prime}} \Gamma_{\left|q^{\prime} \boldsymbol{k}^{\prime} \sigma^{\prime}, \chi^{\prime}\right\rangle}^{|q \boldsymbol{k} \sigma, \chi\rangle}$

It is worth noting that Eqs. (4) and (5) are valid in the nonlinear regime. For our purpose, however, we will linearize these equations with respect to $\delta V$ and $\delta V_{\mathrm{s}}$. Then, the charge and spin currents can be written as [12]:

$$
\begin{aligned}
& I_{\mathrm{c}}=G \delta V+G^{\mathrm{m}} \delta V_{\mathrm{s}}, \\
& I_{\mathrm{s}}=G_{\mathrm{s}}^{\mathrm{m}} \delta V+G_{\mathrm{s}} \delta V_{\mathrm{s}},
\end{aligned}
$$

where $G$ is the charge conductance, $G_{\mathrm{s}}$ can be called spin conductance and describes spin current as a response to spin voltage, while $G^{\mathrm{m}}$ and $G_{\mathrm{s}}^{\mathrm{m}}$ are nondiagonal (mixed) conductances. The later conductances are related via the formula $G_{\mathrm{s}}^{\mathrm{m}}=(\hbar / 2 e) G^{\mathrm{m}}$, which follows from the Onsager relations. Detailed analytical expressions for the above conductances can be obtained from linearization of the expressions for the spin and charge currents [13]. These formula are rather cumbersome and will not be presented here. Instead, we will present some numerical results.

\section{Results and discussion}

Now, we present numerical results on the influence of the magnetic impurity on spin and charge transport. Since $G_{\mathrm{s}}^{\mathrm{m}}=(\hbar / 2 e) G^{\mathrm{m}}$ and $G$ is comparable to $G_{\mathrm{s}}$, we will present in the following only two of them, i.e. the electrical conductance $G$ and the mixed conductance $G^{\mathrm{m}}$. 
In Fig. 1 we show $G$ and $G^{\mathrm{m}}$ as a function of temperature and for indicated values of the parameter $\alpha_{\mathrm{ex}}$ - from $\alpha_{\mathrm{ex}}=0$ (no impurity) to $\alpha_{\mathrm{ex}}=1$ - and for uniaxial magnetic anisotropy $(E=0)$ of easy-axis $(D>0)$ and easyplane $(D<0)$ types. The conductances are normalized there to the corresponding values at $T=0.1 \mathrm{~K}$, where the excited states of the magnetic impurity are not active in transport. For numerical calculations we assumed a magnetic impurity of an integer spin, $S=2$, and also the uniaxial anisotropy constant $|D|=100 \mu \mathrm{eV}$, which is comparable to those observed for single magnetic molecules [6] and also for magnetic adatoms, e.g., Mn [14].
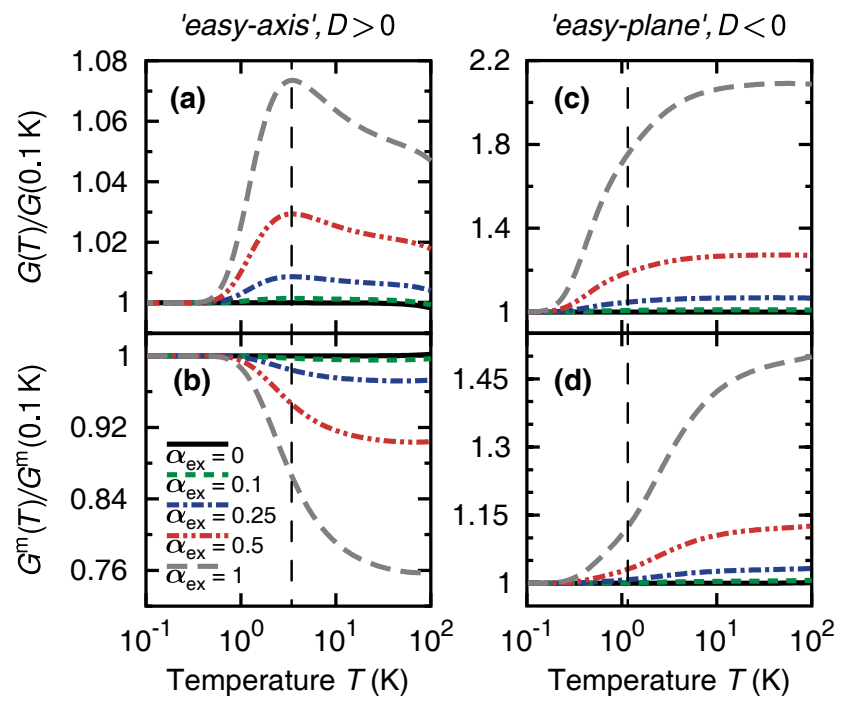

Fig. 1. The effect of a magnetic impurity with $S=2$ on the conductance $G$ and $G^{\mathrm{m}}$ of a magnetic tunnel junction. Dependence of the conductances in the parallel magnetic configuration, normalized to the corresponding values at $T=0.1 \mathrm{~K}$, is shown as a function of temperature for indicated values of $\alpha_{\mathrm{ex}}$ for $D>0$ (a), (b) and $D<0$ (c), (d). Vertical dashed lines represent the temperature equivalent to the zerofield splitting: (a), (b) ZFS $=3 D \approx 3.5 \mathrm{~K}$ and (c), (d) ZFS $=|D| \approx 1.16 \mathrm{~K}$. Other parameters: $K=0.1 \mathrm{eV}$, $\alpha_{\mathrm{d}}=1$ and $p_{L}=p_{R} \equiv p=0.5$.

As one can expect, the effect of excited spin states on transport can be observed only at higher temperatures, $k_{\mathrm{B}} T \gtrsim \mathrm{ZFS}$, where ZFS stands for the zero field splitting, which for $D>0$ is given by $\mathrm{ZFS}_{D>0}=(2 S-1) D$ and for $D<0$ by $\mathrm{ZFS}_{D<0}=|D|$. Indeed, such a behavior is clearly seen in Fig. 1, where the normalized conductance $G$ is constant at low temperature and then grows as soon as the temperature becomes of the order of ZFS (marked by the vertical dashed lines in Fig. 1). Moreover, the increase in conductance is larger for larger $\alpha_{\mathrm{ex}}$, as one could expect. As the electrical conductance $G$ for $D>0$ has a maximum at $k_{\mathrm{B}} T \approx \mathrm{ZFS}_{D>0}$, Fig. 1a, for $D<0$ the conductance $G$ increases monotonously with temperature, Fig. 1c. This behavior can be understood by taking into account temperature dependence of the population of impurity spin states, and also the fact that the lowest state for $D>0$ corresponds to $S_{z}= \pm S$, while for $D<0$ it corresponds to $S_{z}=0$. This difference is also responsible for different behavior of the nondiagonal conductance $G^{\mathrm{m}}$ with increasing $T$, as shown in Fig. 1b and Fig. 1d. However, there is now a qualitative difference, as for $k_{\mathrm{B}} T \gtrsim$ ZFS the conductance $G^{\mathrm{m}}$ grows with increasing temperature for $D<0$ and decreases with increasing $T$ for $D>0$.

The numerical results presented in Fig. 1 are for parallel magnetic configuration. Similar results have been obtained also for the antiparallel magnetic configuration. In the latter case the charge conductance $G$ is smaller, which means that the tunnel magnetoresistance is positive (so-called normal TMR effect). As the magnetoresistance is constant at low temperatures, it becomes reduced for $k_{\mathrm{B}} T \gtrsim$ ZFS, i.e., when the excited spin states of the magnetic impurity start taking part in transport. This behavior is physically reasonable since tunneling processes with spin reversal reduce the spin asymmetry responsible for the TMR effect. Similar behavior can be observed also in the case of the mixed conductance, where the difference in $G^{\mathrm{m}}$ for the parallel and antiparallel configurations diminishes with increasing $T$ for $k_{\mathrm{B}} T \gtrsim \mathrm{ZFS}$.

\section{Summary}

We have analyzed the influence of magnetic impurities embedded in the barrier on spin and charge transport in magnetic tunnel junctions. We have shown that the contribution of electron scattering on the magnetic impurities depends on the type of magnetic anisotropy. Numerical results indicate that the electric conductance increases with temperature when the excited spin states start taking part in transport. This takes place when the thermal energy is comparable or lager than the zerofield-splitting. However, the corresponding tunnel magnetoresistance decreases with increasing temperature for $k_{\mathrm{B}} T \gtrsim$ ZFS. Similar behavior has been also found for the nondiagonal conductance $G^{\mathrm{m}}$.

\section{Acknowledgments}

This work is supported by the National Center of Research and Development in Poland in the frame of EU project Era.Net.Rus "SpinBarrier". M.M. acknowledges support from the Alexander von Humboldt Foundation.

\section{References}

[1] M. Julliere, Phys. Lett. A 54, 225 (1975).

[2] S. Yuasa, T. Nagahama, A. Fukushima, Y. Suzuki, K. Ando, Nature Mater. 3, 868 (2004).

[3] W. Butler, X. Zhang, T. Schulthess, J. MacLaren, Phys. Rev. B 63, 054416 (2001).

[4] S. Yuasa, D.D. Djayaprawira, J. Phys. D Appl. Phys. 40, R337 (2007).

[5] T. Kawahara, K. Ito, R. Takemura, H. Ohno, Microelectron. Reliab. 52, 613 (2012). 
[6] D. Gatteschi, R. Sessoli, J. Villain, Molecular Nano magnets, Oxford University Press, New York 2006.

[7] M. Misiorny, J. Barnaś, Phys. Rev. Lett. 111 046603 (2013).

[8] J. Appelbaum, Phys. Rev. Lett. 17, 91 (1966); Phys. Rev. 154, 633 (1967).

[9] Z. Nussinov, M.F. Crommie, A.V. Balatsky, Phys. Rev. B 68, 085402 (2003).

[10] J. Fransson, Nano Lett. 9, 2414 (2009).
[11] C. Romeike, M.R. Wegewijs, W. Hofstetter, H. Schoeller, Phys. Rev. Lett. 96, 196601 (2006); C. Romeike, M.R. Wegewijs, H. Schoeller, Phys. Rev. Lett. 96, 196805 (2006).

[12] M. Misiorny, J. Barnaś, Phys. Rev. B 89, 235438 (2014).

[13] M. Misiorny, J. Barnaś, Phys. Rev. B 91, 155426 (2015).

[14] S. Loth, K. von Bergmann, M. Ternes, A. Otte, C. Lutz, A. Heinrich, Nature Phys. 6, 340 (2010). 